\title{
Two criteria in Fermat infinite descent method
}

\author{
Anatoly A. Grinberg ${ }^{1 *}$ \\ ${ }^{1}$ (retired) AT\&T Bell Laboratories, Murray Hill, New Jersey, USA \\ *Corresponding author: Anatoly A. Grinberg: anatolyfrgr@gmail.com
}

\section{OPEN ACCESS}

Citation: Grinberg A.A. (2017) Two criteria in Fermat infinite descent method. Open Science Journal 2(4)

Received: $12^{\text {th }}$ September 2017

Accepted: $15^{\text {th }}$ December 2017

Published: $26^{\text {th }}$ December 2017

\section{Copyright:@ 2016 This is an} open access article under the terms of the Creative Commons Attribution License, which permits unrestricted use, distribution, and reproduction in any medium, provided the original author and source are credited.

Funding: The author(s) received no specific funding for this work

Competing Interests: The author have declared that no competing interests exists.

\section{Abstract:}

A method infinite descent is traditionally used to proof the Fermat's theorem for the special case of exponent $n=4$. At each step, the method sequentially generates a new Fermat's equation with one of the term being smaller than that in the preceding step. After a finite number of steps the term becomes less than one and this is taken as criterion of the insolvency of the original Fermat's equation. We show that the power of factor 2 , in even parameter of Pythagoras' equation solution used in the proof, decreases by one at each step of the descent. As a result we arrive at an unsolvable equation. This is the second criterion for the descent method. Which of the two criteria is reached first depends on the parameters of the initial Pythagorean solutions chosen for the analysis.

Keywords: Fermat, Criteria, Infinite, Descent

\section{Introduction}

It is convenient to write Fermat's equation with exponent $n=4$ in the form

$$
\left(\mathbf{X}^{2}\right)^{2}+\left(\mathbf{Y}^{2}\right)^{2}=\left(\mathbf{Z}^{2}\right)^{2}
$$

The variables $\mathrm{X}, \mathrm{Y}, \mathrm{Z}$ are positive, pair-wise relatively prime integers, where $\mathrm{X}$ is even, and $\mathrm{Y}, \mathrm{Z}$ are odd [1-8]. 
Equation (I.1) shows that the squares of these variables, $\boldsymbol{X}^{\mathbf{2}}, \boldsymbol{Y}^{\mathbf{2}}, \boldsymbol{Z}^{\mathbf{2}}$, must coincide with the uniquely defined solutions of Pythagoras' equation, which are determined by the following expressions

$$
X^{2}=2 s u \quad(a) ; \quad Y^{2}=s^{2}-u^{2}(b) ; \quad Z^{2}=s^{2}+u^{2}(c)
$$

where $s$ and $\boldsymbol{u}$ being the odd and the even positive parameters, respectively [4], subject to inequality $\mathrm{s}>\mathrm{u}$ and $(\mathrm{s}, \mathrm{u})=1$. It is evident that solutions for squares $\mathrm{Y}$ and Z, (I.2b) and (I.2c), represent Pythagorean type equations. Therefore, their solutions can also be written in the form (I.2), but with different pairs of parameters.

Despite the fact that the squares of numbers $\mathrm{X}, \mathrm{Y}, \mathrm{Z}$ are integers, the Fermat's theorem states that the square roots of these numbers cannot all be integer at the same time.

Fermat proved the theorem for exponent $n=4$ by the method of infinite descent. At sequential steps of the descent a new Fermat's equation arises with one of the terms smaller than that in the preceding step. After some finite number of steps the term becomes less than one and this is taken as the criterion of insolvency of Fermat' equation in integer numbers.

Below we will show that the exponent of 2 that is factor in the even parameter $u$, decreases by one with each step of the descent. After some number of steps the parameter becomes odd and we arrive at an unsolvable equation. This represents an second criterion for the descent method. Which criterion takes place first depends on the initial values of the parameters $s$ and $u$ in the Pythagorean solutions (I.2).

\section{Proof of the theorem}

Let us write even parameter $\mathrm{u}$ in the form

$$
u=2^{2 k-1} \bar{u}
$$

where $\overline{\boldsymbol{u}}$ is an odd number.

For the solutions of Pythagorean equations, the exponent of 2 in Eq. (II.1) can be any arbitrary positive number. However, due to Eq. (I.2a), it must be an odd number. Indeed, substituting (II.1) into (I.2a)

$$
\mathrm{X}^{2}=2^{2 \mathrm{k}} \mathrm{s} \bar{u}
$$

we se that only for even exponent of 2 can $\mathrm{X}$ be an integer number. Taking into account that $(\mathbf{s}, \overline{\boldsymbol{u}})=1$, the numbers $\mathrm{s}$ and $\overline{\boldsymbol{u}}$ must be squares of some relatively prime odd numbers $\mathrm{p}$ and $\mathrm{q}$, defined as

$$
s=p^{2} ; \bar{u}=q^{2} ; \quad u=2^{2 k-1} q^{2}
$$


Let us substitute (II.3) into (I.2b)

$$
\left.\mathbf{Y}^{2}+\left(2^{2 \mathrm{k}-1} \mathbf{q}^{2}\right)^{2}\right)=\left(\mathbf{p}^{2}\right)^{2}
$$

In Eq. (II.4) all terms are pair-wise relatively prime integers and its solution is

$$
2^{2 k-1} q^{2}=2 s_{1} u_{1}(a) ; \quad Y=s_{1}{ }^{2}-u_{1}{ }^{2} \quad(b) ; p^{2}=s_{1}{ }^{2}+u_{1}{ }^{2}(c)
$$

where $\left(\mathbf{s}_{1}, \mathbf{u}_{1}\right)=\mathbf{1}$ and $\left(\mathbf{s}_{1}, \mathbf{p}\right)=\mathbf{1},\left(\mathbf{u}_{1}, \mathbf{p}\right)=\mathbf{1}$.

Since $\mathrm{q}$ and $\mathbf{s}_{1}$, are odd, the power of 2 in (II.5a) must be equal to (2k-2) and $\mathrm{u}_{1}$ can be written as

$$
\mathrm{u}_{1}=2^{2 \mathrm{k}-2} \bar{u}_{1}
$$

where $\overline{\boldsymbol{u}}_{\mathbf{1}}$ is an odd number. Therefore, similarly to (II.3), it follows from (II.5a) that

$$
s_{1}=p_{1}^{2} ; \bar{u}_{1}=q_{1}^{2} ; u_{1}=2^{2 k-2} q_{1}^{2}
$$

where $\mathbf{p}_{\mathbf{1}}, \mathbf{q}_{\mathbf{1}}$ are odd and $\left(\mathbf{p}_{\mathbf{1}}, \mathbf{q}_{\mathbf{1}}\right)=\mathbf{1}$..

A comparison of Eqs. (II.3) and (II.7) shows that the power of 2 in $\boldsymbol{u}_{\mathbf{1}}$ is less than the power of 2 in $u$ by one. After a finite number of steps of the descent the corresponding even parameter $\mathrm{u}$ becomes odd. Then all three terms of (II.5c)type equation become odd. Clearly, such numbers cannot satisfy the equation. This constitutes the second criterion for the descent method. Either this criterion or the traditional Fermat criterion will determine termination of the descent procedure. The number of steps required until parameter $u$ becomes odd is equal to the power of 2 in expression (II.1). Which of the two criteria will be effective is determined by the values of the parameters $s$ and $u$ in the initial Pythagorean solutions (I.2). Both are required for the consistent application of the descent procedure.

\section{Appendix}

In this section, we follow the procedure of descent for two additional steps, in order to demonstrate the decrease in the exponent of 2 by two more units. Substituting (II.7) into (II.5c) we obtain an equation

$$
\left(2^{2 k-2} q_{1}^{2}\right)^{2}+\left(p_{1}^{2}\right)^{2}=p^{2}
$$

with the solution

$$
2^{2 k-2} q_{1}^{2}=2 s_{2} u_{2}(a) ; \quad p_{1}^{2}=s_{2}^{2}-u_{2}^{2}(b) ; \quad p=s_{2}^{2}+u_{2}^{2}(c)
$$

where $\left(\mathbf{s}_{2}, \mathbf{u}_{2}\right)=\mathbf{1} \quad, \quad\left(\mathbf{s}_{2}, \mathbf{p}_{1}\right)=\mathbf{1} \quad, \quad\left(\mathbf{u}_{2}, \mathbf{p}_{1}\right)=\mathbf{1}$ 
Since $\boldsymbol{q}_{\mathbf{1}}$ and $\boldsymbol{s}_{\mathbf{2}}$ odd, it follows from Eq.(III.2a) that $\boldsymbol{u}_{\mathbf{2}}$ has the form

where $\overline{\boldsymbol{u}}_{\mathbf{2}}$ is odd.

$$
u_{2}=2^{2 \mathrm{k}-3} \bar{u}_{2}
$$

From (III.2a), similar (II.7), we have

$$
s_{2}=p_{2}^{2} ; \bar{u}_{2}=q_{2}^{2} ; u_{2}=2^{2 k-3} \mathbf{q}_{2}^{2}
$$

The equation

$$
\left(2^{2 k-3} q_{2}^{2}\right)^{2}+p_{1}^{2}=\left(p_{2}^{2}\right)^{2}
$$

obtained by substituting (III.4) into (III.2b), has solution

$$
2^{2 k-3} q_{2}^{2}=2 s_{3} u_{3}\left(\text { a) } ; p_{1}=s_{3}^{2}-u_{3}^{2}(b) ; p_{2}^{2}=s_{3}^{2}+u_{3}^{2}(c)\right.
$$

and from (III.6a) it follows that

where $\overline{\boldsymbol{u}}_{\mathbf{3}}$ is odd.

$$
u_{3}=2^{2 k-4} \bar{u}_{3}
$$

We see that the power of 2 in $\overline{\boldsymbol{u}}_{\mathbf{3}}$ is 3 units smaller than in parameter $u$, defined by Eq. (II.1). If $\mathrm{k}=2$ then $\overline{\boldsymbol{u}}_{3}$ becomes odd and equations (III.6b) and (III.6c) are both unsolvable.

\section{Conclusion}

Considering the well-known procedure of infinite descent, we have shown that the power of 2 contained in the even parameters of Pythagorean solutions decreases by one with each step of the descent. Therefore on some steps this parameter becomes odd and we arrive at an unsolvable equation. This is the second criterion of descent method.

The author thanks Prof. S. Luryi for useful discussion.

\section{References:}

1. The 1670 edition of Diophantus' Arithmetica includes Fermat's commentary, particularly his "Last Theorem" (Observatio Domini Petri de Fermat).

2. Bussey W.H. Fermat's Method of Infinite Descent, The Mathematical Association of America, Vol.XXV, No.8, 1918

3. Dickson L.E., "History of the Theory of Numbers", Vol. II, 675 pgs. Carnegie Institute of Washington. Washington 1920.

4. Hinchin A.Y. "Great Fermat's Theorem", 56 pgs. GTTI M - 1934.

5. Postnikov M. M. "Fermat's Theorem", 130 pgs., NAUKA, Moscow 1978.

6. Mishra V.N. Some Problems on Approximations of Functions in Banach Spaces, Ph.D. Thesis (2007), Indian Institute of Technology, Roorkee 247667 Uttaakhand, India.

7. Deepmala, A Study on Fixed Point Theorems for Nonlinear Contractions and its Applications, Ph.D. Thesis (2014), Pt. Ravishankar Shukla University, Raipur 492 010, Chhatisgarh, India. 
8. Mishra L.N. On existence and behavior of solutions to some nonlinear integral equations with Applications, Ph.D. Thesis (2017), National Institute of Technology, Silchar 788 010, Assam, India. 